\title{
Nasal Drops, Solution in Single-dose Container
}

National Cancer Institute

\section{Source}

National Cancer Institute. Nasal Drops, Solution in Single-dose Container. NCI Thesaurus.

Code C149681.

Medicinal product consisting of a nasal drops solution presented in a single-dose container. 